\title{
TRAZANDO EL MAPA DEL PASADO POSTMODERNO (1)
}

\author{
MAPPING THE POSTMODERN PAST
}

\section{IAN HODDER $(*)$}

\section{RESUMEN}

Este artículo empieza describiendo los pasos que deben darse para desarrollar un método de excavación reflexivo en Çatalhöyük. Entre estos 12 pasos, destacamos cuatro temas: relacionalidad (contextualidad) del significado, reflexión (crítica), interactividad y multivocalidad. La idea que subyace en todos ellos es la necesidad de introducir un pensamiento no dicotómico en arqueología. Esta necesidad se basa principalmente en el sistema global moderno (o posmoderno) en el que, cada vez mâs, trabajan los arqueólogos. Está clara la necesidad de fluidez y ruptura de fronteras en un mundo postcolonial inmerso en los sistemas de información.

\begin{abstract}
This paper begins by describing steps that are being taken to develop a reflexive excavation method at Çatalhöyük. From these 12 steps, four themes are described. These are relationality (contextuality) of meaning, reflexivity (critique), interactivity and multivocality. The one idea lying behind all four themes is the need to introduce non-dichotomous thinking in archaeology. The need for such thinking is argued to be especially high in the modern (or postmodern) global systems within which archaeologists increasingly work. The need for fluidity and the breaking down of boundaries is clear in a postcolonial world increasingly linked by information systems.
\end{abstract}

(*) University of Cambridge, Faculty of Archaeology and Anthropology, Downing Street, Cambridge CB2 3DZ, Gran Bretaña.

El artículo fue remitido en su versión final el 26-I-98.

(1) Traducción de Alicia Perea. Dpto. de Prehistoria. Centro de Estudios Históricos. CSIC. Serrano, 13. 28001 Madrid. Revisada por el autor.

Nota de traducción: los neologismos ingleses se han mantenido en español, por ejemplo relacionalidad, contextualidad, especialismos. Entrecomillados y cursivas son del autor, excepto el término latino continuum.
Palabras clave: Reflexión. Globalismo. Multivocalidad. Postcolonialismo.

Key words: Reflexivity. Globalism. Multivocality. Postcolonialism.

\section{HACIA UN PENSAMIENTO NO DICOTÓMICO EN ARQUEOLOGÍA}

En un reciente artículo esbocé la metodología postprocesual que se está utilizando en la excavación del yacimiento de Çatalhöyük en Turquía (Hodder, 1997). Aquí, quiero resumir brevemente los 12 pasos adoptados en ella.

1. Los especialistas del laboratorio visitan las áreas excavadas del yacimiento cada uno o dos días en el transcurso de la excavación. Esto es posible porque cada uno de los expertos en fauna, arqueobotánica, material lítico, cerámico, micromorfología de suelos, geología, restos humanos, y otros están presentes en el yacimiento. El objetivo de las discusiones entre ellos es doble. Desde el punto de vista del personal de laboratorio, se obtiene información sobre el contexto. Por ejemplo, saber si existe alguna incertidumbre en la adscripción estratigráfica o en la datación de un nivel, hogar u otro contexto, ayuda al experto en cerámica. Desde el punto de vista del personal de la excavación, las visitas de los expertos les informan sobre qué es lo que están excavando. Por ejemplo, un especialista en fauna puede identificar sobre el terreno la especie animal y las partes del esqueleto. Esto puede ayudar al excavador a interpretar lo que está excavando y a tomar la decisión correcta en las estrategias de muestreo. Esto nos lleva a la 
segunda parte de la metodología postprocesual de Çatalhöyük.

2. La orientación empiricista y positivista asume la inviolabilidad objetiva de los datos arqueológicos. El resultado es que frecuentemente se desarrollan estrategias de muestreo que puedan aplicarse a una gran variedad de contextos diferentes. Además, el desarrollo de la gestión de recursos culturales ha favorecido la codificación y sistematización de los procedimientos de registro arqueológico. Las estrategias de muestreo se adoptan "listas para llevar", utilizando fórmulas preestablecidas. En la práctica, los arqueólogos tienen el deber de responsabilizarse de lo que encuentren. En consecuencia, estas estrategias se cambian con frecuencia según avanza una investigación o excavación. Pero aún la más codificada de las estrategias de muestreo implica la toma de decisiones interpretativas. Por ejemplo, se pudo haber decidido excavar el $10 \%$ de todas los catas del yacimiento, pero el $20 \%$ de los hogares. Es necesario definir el rasgo que caracteriza a una cata o un hogar antes de la excavación. Pero ¿qué pasaría si se encuentra una nueva categoría, o rasgo, como un hogar ritual? Para evitar estas dificultades en Çatalhöyük hemos sustituido la toma de decisiones sobre el muestreo por acuerdos sobre prioridades. Cuando el personal de laboratorio visita las áreas de excavación, discute con el personal de campo qué niveles y rasgos se van a muestrear con mayor intensidad (cribado con agua por oposición a cribado en seco, por ejemplo). Los porcentajes de depósitos de determinado tipo, a los que se ha dado prioridad, pueden controlarse. Los contextos prioritarios se mantienen en posteriores análisis de laboratorio. De esta manera, el muestreo (priorización) puede relacionarse con la interpretación cambiante del yacimiento y sus correspondientes rasgos. Se puede amoldar a cada yacimiento en particular y adaptarse a su interpretación.

3. Otra característica de la aproximación empiricista y positivista es que asume la naturaleza evidente en sí misma del "objeto arqueológico". Por ejemplo, cuando las cajas de material pasan del yacimiento al laboratorio, generalmente se clasifican como cerámica, metal, hueso, concha, piedra, etc. Esta clasificación determina el modo en que esos objetos van a ser estudiados y publicados. Se envían al especialista en cerámica, metal, hueso etc. Este procedimiento arqueológico tan corriente implica arrancar el artefacto de su contexto. Interpretarlo descontextualizado se hace difícil excepto en términos universales. En Çatalhöyük nos hemos dado cuenta de que este proceso no ayuda a la comprensión del yacimiento o a la de las categorías individuales de objetos. La necesidad del contacto y la integración está detrás de nuestro empeño en contar con la presencia de todo tipo de especialistas en el yacimiento. Pero también nos hemos dado cuenta de que estas categorías son arbitrarias y que dependen de la escala a la que se efectúe el trabajo. A escala microscópica pequeños fragmentos de obsidiana pueden utilizarse como desgrasante de cerámica, de forma que entonces no se clasificarían como "piedra" sino como "cerámica". A gran escala, hemos intentado definir"objetos" que sobrepasen las categorías tradicionales. Por ejemplo, el estudio de los "desperdicios", incluye todo tipo de materiales, lo mismo que "objetos", "quemado", "decoración", "alimento" o "domesticación". De esta manera maximizamos los contactos entre los diferentes especialistas.

4. Otro de los objetivos de las visitas del personal de laboratorio es devolver la información al personal de campo lo antes posible. La razón es combatir la idea de una excavación como proceso mecánico de registrar datos objetivos. Se pretende más bien favorecer la idea de excavación como interpretación "a punta de piqueta". Para la correcta interpretación de una estratigrafía ayuda conocer la datación de la cerámica en cada nivel. Para identificar un suelo podría ser útil saber algo sobre el grado de desgaste de la cerámica y el hueso. De manera que, mientras excavamos, necesitamos saber lo más posible sobre lo que estamos excavando. Este conocimiento, junto con nuestras interpretaciones, será lo que determine la estrategia de muestreo que vamos a emplear. En Çatalhöyük al personal de laboratorio se le pide que siga la pista del material de varios niveles y contextos. En otras palabras, examinan el material rapidamente y reenvian los resultados al personal de campo. Otras formas de acelerar el flujo de información incluyen la digitalización de planimetría y registros, de forma que la distribución de elementos pueda consultarse inmediatamente. Es posible hacer histogramas y comparaciones de forma inmediata para que la excavación se desarrolle con el máximo de conocimiento sobre lo que se descubre.

T. P., 55, n. ${ }^{\circ} 1,1998$ 
5. En un proyecto arqueológico cualquier intento de conectar a los diferentes participantes pasa por una base de datos integral y fluida. En Çatalhöyük hemos trabajado en una red informática de manera que el personal de campo y los especialistas del laboratorio puedan cuestionar sus respectivos datos y comentar las interpretaciones provisionales de cada colega. Todos los tipos de datos, desde el registro de campo, a mapas y dibujos, pasando por medidas de material lítico y cerámico, hasta los diarios y filmaciones que describiremos más adelante, están disponibles en la misma base. Los distintos ordenadores están conectados a un ordenador central al que todo el mundo tiene acceso. El alto grado de interconexión que se consigue significa que las interpretaciones están continuamente en un estado fluido; los "datos" están constantemente reconsiderándose y transformándose, y las conclusiones son efímeras.

6. Por mucho que uno quiera crear una base de datos fluida y flexible algún grado de estabilidad será necesario, de manera que permita comparaciones y el manejo de una gran cantidad de datos. Pero cualquier base es una construcción teórica y es importante que el usuario así lo entienda. El usuario de una base de datos tiene que ser capaz de situarla en su contexto de producción. Para ello, en Çatalhöyük, hemos vuelto a llevar un diario. Se escribe en la base con referencias cruzadas de forma que, si un usuario quiere saber algo sobre el nivel 321, sea posible encontrar todas las entradas referentes al nivel 321 , a la vez que la lista codificada de huesos de animales, cerámica, etc que se encontraron. El diario permite al usuario comprender lo que los excavadores presuponen mientras excavan un nivel determinado. Permite comprender porqué un nivel fue excavado y muestreado de determinada forma. Permite examinar las desviaciones y presunciones. Pero también tiene efectos beneficiosos: otras personas pueden leer las entradas según se introducen de forma que se incrementa el circuito de información. Además, escribir un diario hace reflexionar al excavador sobre el proceso de excavación y evaluarlo en relación a las preguntas que se le están haciendo.

7. Igualmente grabar un video del proceso de excavación conduce a adoptar actitudes reflexivas. En Çatalhöyük las dicusiones del personal de laboratorio durante las visitas al yacimiento (ver punto 1) se graban en video, lo mismo que breves secuencias del trabajo de campo y de laboratorio. Estos videos se digitalizan y editan en clips de $1 \mathrm{o}$ 2 minutos para incluirlos en la base de datos. Son accesibles mediante un sistema de búsqueda con clave. Así, es posible buscar en la base el nivel 321 y encontrar no sólo el objeto, registro de campo y entrada en el diario, sino también el videoclip. Estos clips podrían mostrar al excavador o excavadora del nivel 321 describiendo su trabajo, señalando el nivel y explicando su interpretación. Este proceso facilita la comprensión del usuario de la base mediante una información visualizada. También le permite comprender las presunciones y malos entendidos sobre los que se basó la excavación. De esta forma el "dato" se relativiza dentro de un contexto específico de producción de conocimiento arqueológico. Una vez más, como en el caso del diario, el mismo proceso de filmación significa que la información está circulando entre los miembros del proyecto a la vez que se efectúa su registro y visionado. La reflexión surge cuando se pide a los miembros del proyecto que expliquen su trabajo y sus presupuestos ante la cámara.

8. Ser reflexivo y autocrítico requiere una considerable cantidad de energía y un compromiso con el conocimiento teórico. En la práctica, los arqueólogos puede que tengan escasa inclinación y tiempo para mirarse el ombligo, a pesar de los beneficios que ello reporta. Además, la mayoría no tiene práctica en la observación de comportamientos culturales actuales. Por ello, en Çatalhöyük trabajan con nosotros antropólogos encargados del estudio de la generación de conocimiento. Participan en el día a día del yacimiento, observando y haciendo entrevistas. Uno estudia la forma en que nuestras interpretaciones se imbrican en presiones y presupuestos inconscientes. Otro explora las convenciones visuales mediante las que vemos y registramos el yacimiento (en forma de planos, secciones, dibujos de piezas, fotografías y videoclips). Otro aún estudia el impacto de nuestra presencia en la comunidad local. La presencia de personas cuestionando presupuestos tiene un efecto desestabilizador en los equipos de excavación e investigación. Pero se necesita una cierta falta de estabilidad si aspiramos a una aproximación crítica y si el proyecto va a responder al mundo cambiante que le rodea.

9. Se han tomado medidas para poner en la Web toda la base de datos de Çatalhöyük de mane- 
ra que se facilite al máximo la participación en la interpretación del yacimiento por parte de una variedad de grupos diferentes. El objetivo es ofrecer una base accesible y multimedia. Este tipo de apertura puede entrar en conflicto con los intereses individuales y de grupo con acceso especial al yacimiento. Por ejemplo, la trayectoria profesional de los miembros más jóvenes del proyecto puede verse amenazada si otros tienen acceso a, y publican, datos inéditos. Desde luego, es factible la aparición de sitios Web alternativos sobre Çatalhöyük por parte de grupos de la competencia. Sin embargo, aunque los derechos de grupos e individuos deban ser protegidos, ello no puede justificar el secretismo de la información arqueológica a largo plazo. La accesibilidad inmediata favorece la participación y el compromiso con el propio proceso de investigación. Incrementa la multivocalidad.

10. La linearidad del relato arqueológico limita la complejidad de las historias que se pueden contar. También fomenta la disociación entre evidencia e interpretación. Esta última suele presentarse después de haber expuesto la evidencia. El hipertexto, por el contrario, permite relaciones de vías múltiples e incorporar los multimedia. Así, se puede ofrecer una relación narrada y facilitar conexiones entre la narración y las fotos, los planos y los datos codificados del material. El usuario del hipertexto puede accionar el ordenador e ir del texto narrativo a la evidencia del dato para comprobar las bases sobre las que se han elaborado esas interpretaciones.

11. Los arqueólogos han hecho siempre planos, dibujos y modelos de los edificios que excavan. Estas y otras reconstrucciones permiten plantear hipótesis para la experimentación sobre técnicas constructivas originales. También permiten la participación de un público más amplio en la comprensión del yacimiento. Actualmente las técnicas de Realidad Virtual dan mayor rapidez y flexibilidad a la experimentación de reconstrucciones. La construcción de un mundo virtual en el ordenador permite la visualización y la experimentación con reconstrucciones alternativas. Igualmente, el mundo virtual puede ser interactivo de manera que el usuario pueda hacer preguntas sobre el yacimiento y explorarlo desde un punto de vista no especializado. En Çatalhöyük queremos que la reconstrucción virtual del yacimiento sea la "cabecera" de la base de datos. Los usua- rios no especialistas pueden así "navegar" por el yacimiento virtual y encontrar la información arqueológica con el nivel de detalle deseado. La virtualidad permite también experimentar con las diferentes maneras en las que se tiene experiencia del yacimiento.

12. En Çatalhöyük se anima a equipos de diferentes partes del mundo a que excaven sus propias zonas en el yacimiento. Se utilizan sistemas de documentación y recogida de datos equivalentes, pero cada equipo utiliza sus propias técnicas de excavación y análisis tradicionales. Se presupone que los distintos equipos, utilizando diferentes métodos, producirán diferentes resultados. $\mathrm{Al}$ mirar por ventanas distintas, cada equipo verá y encontrará diferentes Çatalhöyüks. Esta diversidad, más que censurarla como caótica, es bienvenida porque es preferible a un acercamiento monolítico y de perspectiva única. Esta última produciría una relación coherente, pero esa relación estaría basada en presupuestos de cada tradición arqueológica que se dan por sentados.

Parece que hay cuatro temas subyacentes en los doce pasos prácticos de una excavación descritos más arriba: reflexión, relacionalidad o contextualidad, interacción y multivocalidad.

Reflexión. Con ello me refiero a la observación de los efectos que nuestras acciones y supuestos tienen sobre los diferentes grupos implicados en un proceso arqueológico, desde los demás arqueólogos hasta la comunidad local. Un ejemplo de nuestro empeño en Çatalhöyük es el trabajo de los antropólogos que incluye el impacto del proyecto en la comunidad local, así como en los grupos nacionales e internacionales que están interesados o visitan el yacimiento. La reflexión se induce igualmente a través de la escritura del diario y la filmación de videos, puesto que estos procesos invitan a los componentes del equipo a estudiar sus propias presunciones. Los diarios y videos proporcionan también información contextual del proceso de excavación, de manera que otros puedan volver a evaluar críticamente las declaraciones realizadas. Los resultados se relacionan reflexivamente con el contexto en el que se genera el conocimiento.

Relacionalidad y contextualidad. La idea aquí es que el significado es relacional. Este empeño se

T. P., 55, n. ${ }^{\circ} 1,1998$ 
ve en el intento reflexivo de relacionar los hallazgos con un contexto específico de producción de conocimiento. Pero también se hace patente en las interrelaciones de la información artefactual y contextual.Así, la datación de un nivel depende de los objetos encontrados. Pero en ocasiones, la datación de los objetos puede depender de las relaciones estratigráficas de los niveles. En otro ejemplo de Çatalhöyük la interpretación de un edificio como habitación, más que como capilla, depende de los objetos en su interior; pero la interpretación de los objetos depende en parte de si la edificación se ve como habitación o como capilla. De manera que generalmente, en arqueología, todo depende de todo en una totalidad hermenéutica. Nuestra intención en Çatalhöyük ha sido facilitar esta interconexión, por ejemplo teniendo disponible para los excavadores la información sobre los objetos mientras excavan un contexto en una cuadrícula. La interpretación del objeto y del contexto dependen uno del otro, de manera que es necesario disponer en el propio yacimiento de muchos especialistas en contextos y objetos para que la información sea accesible para cada cual, especialmente para los propios excavadores. La finalidad está en conseguir una alta integración e interdisciplinareidad. La relacionalidad implica también flexibilidad en el proceso de investigación. Si todo depende de todo y yo cambio una variable en mi análisis, habrá efectos incontrolables en todas las demás. Por eso la base de datos de Çatalhöyük es tan flexible y cambiante como sea posible; las conclusiones son siempre provisionales y objeto de transformación.

Interacción. La intención aquí es proporcionar mecanismos para que la gente pregunte y critique las interpretaciones que se están haciendo, en el momento en que se hacen. Se estimula la interacción entre el personal de laboratorio y el de campo durante la excavación con las visitas a los cortes. A los procedimientos de priorización (muestreo) se llega mediante negociación entre los miembros del equipo. También se facilita la interacción en Çatalhöyük disponiendo la base de datos en la Web y dando las rutas de acceso de forma amigable (por ej. reconstrucciones virtuales). También se facilita suministrando la información en forma de diarios y videos que disponen y abren la base de datos a la crítica y a interpretaciones alternativas. El objetivo es tener una sección para la comunidad en el Museo del yacimiento donde lo que se muestre haya sido elaborado por miembros del pueblo cercano. En el Museo también habrá un CD-Rom interactivo, con hipertexto y componentes de realidad virtual, para que los visitantes y estudiantes puedan aprender del yacimiento de forma no lineal.

Multivocalidad. Una amplia gama de grupos distintos tiene, frecuentemente, intereses que entran en conflicto y quieren involucrarse en el proceso arqueológico de diferentes maneras. Hay que proporcionar mecanismos para que cada discurso pueda expresarse. Por ejemplo, en Çatalhöyük distintos equipos excavaron distintas zonas del yacimiento y propusieron sus propias visiones. Mientras que el sitio Web permite la comunicación con otras redes de grupos internacionales de cierto nivel intelectual, la comunidad local rural puede contactar mejor a través de las exposiciones del museo y las visitas al propio yacimiento. En el futuro puede que haya grupos como el de la Diosa Madre que quieran rezar en el yacimiento.

En términos generales, se puede argumentar que hay un tema subyacente en estos cuatro puntos de interés y es la ruptura de límites y dicotomías. Los arqueólogos han pasado mucho tiempo a lo largo de su historia poniendo en orden los límites de su disciplina. Tenían que defenderlos de los anticuarios, de los clandestinos. Creacionistas, usuarios de detectores de metales, movimientos de reinhumadores, adoradores de la Diosa. Algunos de estos grupos han sido calificados de "marginales". Otros están completamente fuera de la disciplina, pero al final, el mantenimiento estricto de esos límites, aunque eficaz en algunas circunstancias, restringe la posibilidad de diálogo y compromiso. Por ejemplo, muchos arqueólogos, al menos en Gran Bretaña, se dan cuenta ahora de que pueden colaborar con usuarios de detectores de metales en un marco que implica educación y mutua comprensión. El conflicto de los reinhumadores en Australia y Estados Unidos ha supuesto un amplio toma y daca por ambas partes. Allí donde se desarrolla una arqueología indígena, al menos en la etapa postcolonial, existe algún tipo de negociación. Allí donde se necesita al mercado como soporte financiero para la investigación arqueológica, se deben prever orientaciones para un público diverso. De manera creciente, la arqueología se ha dado cuenta que necesita incorporar una diversidad de orientaciones y esta- 
blecer una diversidad de mecanismos para involucrarse con el pasado.

Hay que romper también con los límites en torno a los especialismos en arqueología. Según ha ido madurando, la arqueología se ha diversificado con éxito y han ido surgiendo una amplia gama de especialismos. Esto fue necesario y sano, pero ahora se necesita una integración y una investigación que rompa con los límites de las especialidades. Me he referido más arriba a la necesidad de relacionalidad e interactividad en el proceso arqueológico. Tenemos que identificar nuevos objetos de estudio que superen los límites del especialismo. A una mayor integración se dirigen las nuevas técnicas de información.

Tenemos que romper las barreras en torno al yacimiento. Esto es en parte una cuestión de abrirlo a una gama más amplia de visitantes y de fomentar la interacción y multivocalidad. Pero también es cuestión de reconocer los efectos radicales de las nuevas tecnologías de la información. Interesa saber que en el discurso de la globalizadora jerga Web, el término "sitio" (2) se ha escogido para significar un lugar o dirección de la World Wide Web. Sería posible construir nuevos lugares Web que actuaran de alternativa a los "sitios" oficiales. Por ejemplo, hay muchos sitios Web en los que se puede encontrar información sobre el "sitio" (arqueológico) de Çatalhöyük. De alguna manera, el único lugar donde Çatalhöyük no existe es en Çatalhöyük. Quiero decir que la gente construye sus propias versiones de Çatalhöyük. Lo pueden hacer en un sitio Web o por otros medios, o incluso en sus mentes e imaginación. Cuando vienen al yacimiento, lo ven desde su propia perspectiva, de la misma forma que cada equipo que trabaja allí construye su propia orientación. Todas estas interpretaciones variables del yacimiento arqueológico son deudoras de otros "sitios" (otros lugares, otros sitios Web, imaginaciones). La arqueología tiene que ser multi-sitio. Tiene que ser abierta y menos restringida.

Como corolario del último punto, es necesario romper los límites alrededor del equipo. Según se va involucrando más y más gente en la excavación, análisis e interpretación de un yacimiento arqueológico, el equipo que está trabajando allí se vuelve muy difuso, abierto y flexible. En Çatal-

(2) El autor juega aquí con el término inglés "site" que tiene tres acepciones: yacimiento (arqueológico), sitio o lugar, y lugar web.

T. P., 55, n. ${ }^{\circ} 1,1998$ höyük yo he tenido cada vez mạ́s dificultades en saber claramente quien es y quien no es miembro del equipo. Algunos ni siquiera han estado nunca en el yacimiento (excepto en el sitio Web) (2). Otros han visitado el yacimiento y colaborado pero no podrían calificarse de arqueólogos. Los comentarios que los extraños hacen en la Web han resultado muy útiles y se han incorporado al proceso de investigación de los "equipos". Los cuatro temas identificados más arriba, pero particularmente la interacción y multivocalidad, implican el emborronamiento de los límites de cada equipo particular.

Los límites en torno al autor se han vuelto igualmente borrosos. Al tiempo que se involucran más voces en el proceso arqueológico, se fomenta la interacción y se adopta una postura de autorreflexión crítica, igualmente se va diluyendo la distintiva voz del autor único. Al tiempo que el estatus y autoridad del autor se abren a la evaluación crítica y se introducen múltiples medios de comunicación, igualmente disminuye el lugar privilegiado del mundo escrito. En la medida en que se incrementa la utilización del hipertexto, la linealidad del texto escrito y sus recurso retóricos específicos son reemplazados por flujos de información multicanales y multinodales. El autor desaparece en un flujo de información, de signos que se reemplazan unos a otros en una red abierta.

Al tiempo que estos límites se vienen abajo, lo mismo ocurre con las principales dicotomías que sustentaron la arqueología desde sus inicios. Romper los límites que encorsetaban la disciplina y los especialismos significa romper la dicotomía entre ciencias y humanidades. La arqueología no es una ciencia o una humanidad. Es ambas cosas a la vez. El proceso arqueológico implica una gran dependencia de las ciencias naturales para la datación, el estudio de los procesos de formación del yacimiento, el análisis del cambio del medio ambiente, la procedencia de los objetos intercambiados, etc. Tal información debe situarse dentro del marco de conocimiento de las humanidades ( $\mathrm{vg}$. historia) y ciencias sociales ( $\mathrm{vg}$. antropología social y cultural) sobre la organización social y la manipulación de la cultura. Desde luego, el término cultura material, principal interés del cuestionamiento arqueológico, encierra la dualidad inherente a la disciplina. La arqueología depende del estudio científico de los materiales para inferir patrones culturales. 
Las causas de la variabilidad en el registro arqueológico no son culturales o naturales. Son ambas cosas. Muchos arqueólogos aceptarían hoy día que mientras el medio y las fuerzas materiales condicionan la iniciativa del hombre, el carácter específico del comportamiento humano está igualmente informado por la elección cultural y la intencionalidad. A esta visión dialéctica se ha llegado desde muchas direcciones. La vemos en una generación anterior, en el trabajo de Grahame Clark (1957: 219) cuando argumenta que mientras el medio limita o condiciona la elección social, esta última determina el comportamiento cultural. La vemos en la discusión neomarxista sobre la relación entre fuerzas y relaciones sociales de producción (Friedman y Rowlands, 1978; McGuire, 1992). La vemos en las actuales aplicaciones de los sistemas complejos (Flannery y Marcus, 1976; 1993; van der Leeuw y Torrence, 1989) y en las aproximaciones fenomenológicas a paisajes y monumentos (Thomas, 1996; Tilley, 1994). En todos estos casos se intenta de diferentes maneras pensar sobre la naturaleza y la cultura no como "esto y lo otro" sino en términos de "ambas".

Pero quizá la mayor dicotomía que envició a la arqueología haya sido la separación cartesiana entre sujeto y objeto (Knapp y Meskell, 1997). A los arqueólogos siempre les ha interesado distinguir entre un pasado subjetivo y otro objetivo. Las visiones subjetivas tienen que ser contrastadas con datos objetivos. Pero incluso los arqueólogos procesualistas han tenido que aceptar que los datos arqueológicos están informados por la teoría (Renfrew y Bahn, 1991) y que teoría y datos se relacionan dialécticamente (dependen una del otro en un circuito teoría-dato). Esta visión se articula más claramente aún en la arqueología neomarxista (v.g. Friedman y Rowlands, 1978; McGuire, 1992) y postprocesual (Hodder, 1982). Si el proceso arqueológico está abierto a la interacción y la multivocalidad; si los límites en torno a la disciplina, yacimiento, equipo y autor se han roto, entonces no puede ser adecuado por más tiempo separar un pasado objetivo, definido por los arqueólogos, y un pasado subjetivo, definido por no arqueólogos. Todos interpretamos el pasado desde perspectivas diferentes y estas distintas interpretaciones pueden contrastarse con la evidencia. Se pueden comparar distintas teorías utilizando una variedad de mecanismos. El pasado no es objetivo o subjetivo. Es ambas cosas. Con esto quiero decir que la evidencia arqueológica tiene una materialización "objetiva" que limita y confronta lo que se puede decir sobre él, y que contribuye a la experiencia de los observadores "subjetivos". Al mismo tiempo, el intérprete "subjetivo" de la evidencia construye los datos "objetivos" desde una perspectiva particular.

\section{FLUJOS DE INFORMACIÓN GLOBAL}

He descrito más arriba un proceso arqueológico que es reflexivo, relacional, interactivo y multivocal. El propósito es acabar con los límites entre el interior y el exterior de la disciplina, entre los diferentes especialismos, entre yacimiento y no yacimiento, entre autor y lector, entre sujeto y objeto, etc. La ruptura de estos límites estimulan el intento de pensar en términos no dicotómicos, esto es, en términos de "ambos/y" mejor que "uno u otro". Al apartarnos de las dicotomías buscamos metáforas alternativas. Más que límites, describimos redes y flujos. Más que oposiciones rígidas, percibimos fluidez. Más que espacio y tiempo, intentamos resolver el espacio-temporalidad.

Me gustaría demostrar que la búsqueda de un discurso más incluyente en arqueología no debiera organizarse como un orden del día preceptivo; la búsqueda forma parte, más bien, de los grandes cambios de la sociedad hacia redes y flujos (Castells, 1996). Estos cambios se califican de alto o post-modernismo, era de la información, sociedad de consumo, sociedad postindustrial, sociedad mediática, etc. Es importante comprender la naturaleza y dirección de estos cambios si queremos dar sentido al impulso hacia un pensamiento no dicotómico en arqueología.

Demostraré aquí que las tendencias que nos impelen hacia una sociedad de redes se comprenden mejor en el contexto de la globalización, y que la globalización presenta los tres componentes que se reflejan en la figura 1 . En el vértice están las tendencias homogenizadoras asociadas obvia y fácilmente con Internet y las superautopistas de la información. La dispersión de los modos de trabajo y la común preocupación por el medio ambiente también producen homogenización. El resultado final es una pérdida de la diversidad cultural al tiempo que surgen McDonalds y Coca-Cola como partes de la "aldea global" y de un mercado homogéneo. Los gobiernos se vuelven serviles con las compañías multinacionales.

T. P., 55, n. ${ }^{\circ} 1,1998$ 
Pero uno de los atractivos del proceso globalizador es que genera diversidad y fragmentación tanto como homogeneidad. Una vez más, necesitamos un pensamiento no dicotómico. En la figura 1 aparecen dos procesos de fragmentación bastante diferentes: la fragmentación producida por el proceso de un capitalismo avanzado, y la búsqueda de identidad dentro y contra el mercado.

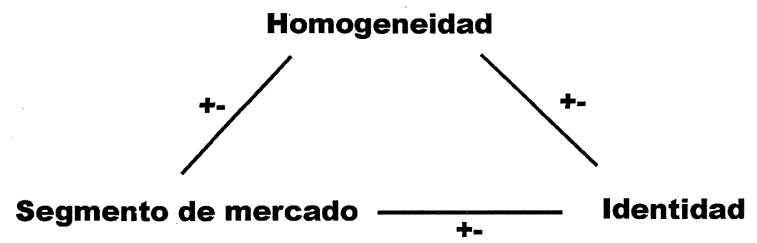

Fig. 1. Homogeneidad. Segmento de mercado. Identidad. Elementos contrapuestos del globalismo.

La fragmentación la produce el mercado de formas distintas, en el marco del capitalismo tardío y avanzado. Por ejemplo, cambios en los modos de trabajo producen regimenes flexibles según los cuales la gente trabaja ocasionalmente o en casa. También producen fragmentación las nuevas tecnologías de la información. La televisión, etc., pero además los VCR, walkmen, y la cantidad de canales disponibles, todo conduce a una gran cantidad de alternativas individuales de elección; hay descentralización y mercantilización. Es incluso posible para los gestores de laWeb adaptar la oferta de información a medida de cada uno. Se ha hecho posible el mercado de nicho e incluso el mercado individualizado.

Al mismo tiempo, los individuos pueden utilizar estas mismas tecnologías para crear nuevas comunidades y nuevas identidades. Internet ha permitido la proliferación de grupos de interés específicos. La sociedad postmoderna ha visto surgir pequeños grupos que pueden ser localizados o estar dispersos (comunidades virtuales). Pueden ir en contra de la cultura de masas o favorecerla. Pueden formar parte del mercado global o ser su antítesis. Igualmente existen aquellos que se sitúan fuera de la red global, cuyas identidades están conectadas a un lugar y a la historia marginal. Son los menos ricos, los menos educados, los menos conectados. Tales comunidades reciben muy poco de la cultura global; su explotación está asociada a la alienación y la resistencia (Fig. 1).

T. P., 55, n. ${ }^{\circ} 1,1998$

\section{TRAZANDO EL MAPA DEL PATRIMONIO GLOBAL}

Quiero ahora trazar el mapa, primero del patrimonio y despues de la arqueología dentro del esquema planteado en la figura 1. El patrimonio se ha convertido en una industria global que crece a buen ritmo. Está implicado en todo el contradictorio proceso descrito en la figura 1, tal y como se indica en la figura 2.

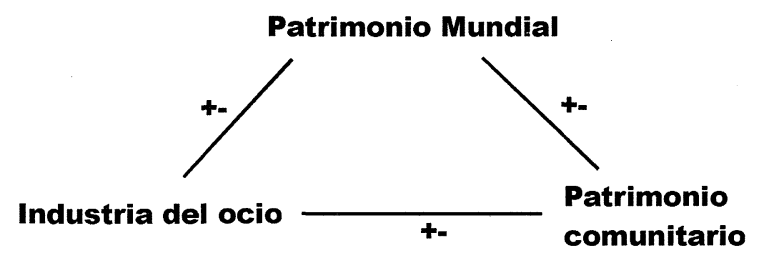

Fig. 2. Patrimonio mundial. Industria del ocio. Patrimonio comunitario. Elementos contrapuestos de patrimonio global.

La homogenización del patrimonio se hizo patente con el surgimiento del concepto de «patrimonio mundial» y la designación de lugares como tal patrimonio por la UNESCO en las últimas décadas. La cuestión subyacente es proteger aquellos sitios de importancia global y que forman parte de una «comunidad humana». La intención es proteger para todos «nuestro» patrimonio común. A una escala diferente, la Comisión Europea nombra lugares de interés para Europa como una totalidad, más que para determinados estados-nación.

Este proceso homogenizador tiene relaciones positivas y negativas con el mercado. Uno de los efectos de la designación de un yacimiento como patrimonio mundial o europeo puede ser la explotación comercial. La visibilidad internacional que confiere a un yacimiento tal designación puede atraer al turismo y a la industria del ocio. Viajar a otro tiempo, lo mismo da en coches del tiempo de Disneylandia que como parte de un tour internacional, constituye el punto central de la industria del ocio, un componente esencial de lo exótico de la alteridad hacia la que parece nos sentimos más atraidos cada vez. Como lo describe Lowenthal (1985), el pasado se ha convertido en otro país al que queremos huir como parte de nuestro viaje de ocio. El resultado para los yacimientos puede ser una inyección de fondos y la capacidad de los grupos locales o gobiernos para añadir infraestructu- 
ra. El turismo puede, además, elevar la riqueza y la oferta de empleo local.

Pero también existen tensiones entre el patrimonio mundial y el mercado. Por ejemplo, a las agencias internacionales les corresponde proteger los yacimiento del saqueo y vandalismo. El desarrollo comercial para turistas puede beneficiar más a las compañías internacionales que a la comunidad local. Se puede forzar este desarrollo (hoteles, tiendas, aparcamientos) por los intereses creados en el yacimiento.

De igual forma, existen relaciones positivas y negativas entre el patrimonio mundial y la formación de identidades comunitarias. La atención internacional y la influencia del turismo pueden aumentar el sentido local de identidad. La formación de nuevas comunidades puede surgir de la conciencia renovada de un patrimonio común. Estas comunidades y sus tradiciones pueden «inventarse», como en el caso de la identidad pan-nativa americana. También pueden estar dispersas, como el caso del movimiento de la Diosa en el que se implican mujeres a la búsqueda de un mundo original anterior al patriarcado. Locales o dispersas (virtuales), se ven fortalecidas al pertenecer a redes globales. Lo mismo que en el caso del Tribunal Europeo de Derechos Humanos o los Tribunales Internacionales de Guerra pueden proteger al individuo contra el Estado, así el estatus de Patrimonio Mundial protege yacimientos y llama la atención sobre las identidades locales. Los contactos globales promovidos por el Congreso Arqueológico Mundial ayudan a las identidades emergentes basadas en el patrimonio (Ucko, 1987).

Los procesos globalizadores suponen mecanismos de emancipación, pero también crean mayores posibilidades para la dominación y la exclusión. Algunos gobiernos evitan las designaciones de patrimonio mundial porque permiten la intervención de agencias internacionales en los programas de patrimonio nacional. Tanto el estatus de patrimonio mundial como la explotación comercial pueden llevar a un yacimiento a desgajarse del significado y la identidad locales. Los oriundos pueden ver negado o interrumpido el significado tradicional de esos yacimientos por agencias externas. El patrimonio de identidades locales puede volverse fragmentario fácilmente a través del proceso globalizador.

Los grupos e individuos que no pertenezcan a una red se apartan de la información global y tienen dificultades para el acceso al conocimiento sobre patrimonio. Pueden hablar lenguas que los excluyan del debate internacional (por ej. Olsen, 1991). La multivocalidad y apertura sólo se da entre los que pertenecen a una red. El resultado puede silenciar otras voces e impedir la última oportunidad de incorporación a la homogenización global. Como alternativa, el que no pertenece a una red puede formar identidades culturales y patrimoniales de forma tradicional, reaccionando contra las nuevas formas de comunidad virtual.

Igualmente existen relaciones positivas y negativas entre patrimonios comunitarios y la industria del ocio. Como ya se ha señalado, el turismo puede promocionar el crecimiento y el empleo de comunidades locales y promover identidades comunitarias mediante la gestión del patrimonio. Se pueden citar casos como el de los Hopi-Tewa en el suroeste americano, donde la explotación comercial de la artesanía tradicional contribuyó a la producción y el empleo locales. El compromiso con «otras» formas de vivir por parte de los turistas y visitantes tiene igualmente efectos «beneficiosos» para los países desarrollados. La nostalgia y sensación de escapismo que descubre la gente puede ayudarles a superar la alienación y la fragmentación de la vida moderna. De esta forma quedan satisfechos los intereses del capitalismo avanzado y del mercado.

La relación entre mercado y patrimonio comunitario puede ser también negativa. Los «grandes negocios» pueden hundir fácilmente los intereses locales y abolir sus derechos. La comercialización puede convertir la identidad en un pastiche, la continuidad en fragmentación. Pasados al estilo Disney pueden trivializar reivindicaciones y sensibilidades. La idea de un «Parque temático de Auschwitz» dramatiza la insensibilidad a la que se puede llegar. ¿Cómo es posible que algunos sufran en un lugar mientras otros disfrutan? Tales ejemplos y tensiones están bien definidos. En otros casos, las líneas entre el pasado como ocio y el pasado como identidad comunitaria son más difíciles de trazar. Por ejemplo, entre los viajeros que vienen a Avebury y Stonehenge en Gran Bretaña a celebrar el solsticio de verano, hay grupos como los Druidas y los Anarquistas de Wessex. ¿Se dedican a disfrutar o a la acción política y social? ¿Están estos grupos igualmente comprometidos con una causa social? ¿Dónde está la línea que separa el juego de la pasión? Y aún si la gente estuviera comprometida pasional- 
mente con una determinada causa, ¿es su compromiso resultado de la diversidad generada por el capitalismo avanzado? Las respuestas a estas cuestiones son difíciles. Una vez más, como en las relaciones de la figura 2 , el nexo entre mercado, incluyendo la industria del ocio, y patrimonio comunitario es dialéctica y no dicotómica.

\section{TRAZANDO EL MAPA DE LA ARQUEOLOGÍA GLOBAL}

La homogenización diseñada por los procesos de globalización puede compararse con la búsqueda de leyes y métodos universales, aplicables sin tener en cuenta el momento o el lugar, de la arqueología de fines del siglo XX. Claramente este fue el grito de guerra de la arqueología procesual. Aunque en la arqueología postprocesual se pone un menor énfasis en los universales, no se puede negar que se hacen afirmaciones preestablecidas de manera rutinaria, tales como "la cultura material tiene significado", "el registro arqueológico es (o no es) un texto", "la estructura es el medio y resultado de la acción". Aunque los arqueólogos postprocesuales (y neo-Darwinianos) intentan retomar la historia, la contingencia y la indeterminación, sin embargo, sus argumentos para la diversidad y la diferencia se basan en afirmaciones generales.Así, todas las arqueologías, sea cual sea su tradición, se valoran en términos de esos argumentos generalizadores.

Las tendencias universalistas de la arqueología angloamericana pueden conducir claramente a la desaparición de la historia. Trigger (1984) ha insistido en que el estudio de los nativos americanos por parte de la arqueología procesual condujo a la búsqueda de generalizaciones tipo ley y a una trivialización de la prehistoria específica de los grupos nativos americanos como una finalidad en sí misma. Argumenta que al negar la validez del estudio de la prehistoria de zonas específicas del mundo, los nuevos arqueólogos afirmaron la escasa importancia de las tradiciones nacionales propias y de cualquiera que estuviera en el camino de la actividad económica americana y de su influencia política. Con anterioridad, el punto de vista colonial condujo al establecimiento de métodos arqueológicos británicos en India y Australia, por ejemplo (Clark, 1957). Desde luego, allí donde se practique la arqueología hoy, sus métodos están influidos por, si no son totalmente de- pendientes de los métodos y perspectivas euroamericanos.

En algunas regiones, especialmente aquellas con una historia reciente de dictadura, como España o Chile, los argumentos universalistas y los métodos objetivos pueden sentar unas bases para la crítica de las estructuras de autoridad establecidas. Proporcionan una vía para sacar a la luz las usurpaciones arbitrarias del poder. Promueven la democratización. En otras regiones, la ciencia objetiva se asocia ella misma con el ejercicio arbitrario de un poder centralizado. Por ejemplo, en el conflicto sobre la reinhumación de los restos de nativos americanos o la de los derechos sobre la tierra de los aborígenes australianos, la ciencia arqueológica objetiva se asoció con los intereses del poder establecido, contra el que tenían que luchar las comunidades locales. Desde luego, en el mundo postcolonial la investigación y administración arqueølógicas se han ido haciendo cada vez más "indigenistas". Aunque el cambio a manos de los locales de su propio pasado pudo promover la escritura de pasados contextualizados, los métodos utilizados procedían a menudo del legado colonial. Por ejemplo, los arqueólogos que trabajan en los Andes peruanos podrían llevar a cabo sacrificios rituales aymara cuando violan los enterramientos o abren catas en los antiguos lugares. El debate global se efectúa en los términos establecidos por el centro dominante. Las redes globales de información producen prácticamente el mismo efecto.

La amplia disponibilidad de información arqueológica en la Web puede socavar la identidad de las comunidades locales y diluir su carácter distintivo. La trayectoria profesional de un individuo puede verse amenazada -por ejemplo, investigadores cuyas carreras dependen de la publicación de datos inéditos de un yacimiento pueden rechazar que "sus" datos estén al alcance de todo el mundo. Pero igualmente la disponibilidad de información en la Web puede permitir a alguna comunidad reafirmar su identidad. Personas desperdigadas por el globo pueden acceder al dato arqueológico para su investigación o por el interés político o personal. Por ejemplo, los grupos de la Diosa podrían aprender de los trabajos de María Gimbutas (Meskell, 1995). Este último proceso puede tener como resultado una erudición superficial cercana al juego (ibid.), o simplemente puede atraer a los grupos locales al discurso dominante. Pero también existe la posibilidad de una formula- 
ción de perspectivas alternativas. Por ejemplo, Mamani Condor (1989) defendió una orientación de la arqueología desde perspectivas indígenas con respecto a los monumentos como partes «vivas» del paisaje. En India, Paranjpe (1990) ha empezado a buscar otras orientaciones, en contra de los presupuestos universalistas de los intelectuales occidentales (ver también Ucko, 1995). Estos opinan que las teorías indias sobre significado e interpretación deben tomarse como una reacción contra el discurso occidental.

Los arqueólogos se están dando cuenta cada vez más de que los informes de su investigación tienen que escribirse a menudo en términos muy distintos, para una gran variedad de audiencias. El límite entre un contenido universalista o contextualizado, por un lado, y la versión popularizada del otro, es a menudo difícil de establecer. Y es cierto que, como en la mayor parte del mundo postmoderno, la frontera entre «alta» $\mathrm{y}$ «baja» cultura debe difuminarse. Muchos de los asuntos más conocidos tienen efectos sociales importantes, como en las publicaciones para niños o en las presentaciones para una amplia audiencia de la televisión. Pero, como ya se advirtió, los intelectuales pueden ser tergiversados y malinterpretados fácilmente. El predominio cada vez mayor del patrocinio comercial de los proyectos arqueológicos plantea la posibilidad de un sesgo hacia «lo que el patrocinador quiere» al escribir los informes. A menudo los arqueólogos exageran la importancia de los hallazgos para satisfacer la necesidad de publicidad y sensacionalismo del patrocinador. Comercio y ocio pueden llegar a comprometer así la profesionalidad y metodología del arqueólogo. Por otra parte, no cabe duda de que la mayor parte de la arqueología actual, sobre todo en Estados Unidos y Gran Bretaña, depende de los patrocinadores. Las relaciones con patrocinadores corporativos se pueden encauzar y dirigir de tal manera que se minimicen las interferencias en beneficio de las dos partes.

Quizá la mayor conexión entre comercio y arqueología se dé en aquellos países que han establecido un sistema de gestión de los recursos culturales financiado por expertos en desarrollo. En estos casos, sin duda existen tensiones (Hunter y Ralston, 1993). Los equipos arqueológicos pueden conseguir puestos de trabajo bajando los costes a su nivel mínimo. Puede que se invierta muy poco en investigación y publicación. El trabajo puede ser a corto plazo e inseguro. Puede que haya oportunidades muy limitadas para el desarrollo de planes de investigación integrados y estrategias regionales. El crecimiento de una arqueología financiada comercialmente ha llevado a establecer una serie de normas de codificación de procedimientos y de seguimiento de resultados por las agencias centrales. De este modo han surgido tendencias universalistas.

La gestión de recursos culturales ha permitido ciertamente la expansión de la arqueología a gran escala en determinados países. Pero estos efectos beneficiosos se ven neutralizados por las tendencias hacia la codificación y regulación. Esto contradice precisamente la apertura y multivocalidad de una orientación incluyente hacia el pasado. Contradice directamente los sistemas fluidos, los métodos integradores y contingentes identificados al principio de este artículo. Impide el reconocimiento de otras voces e intereses. Como resultado, en muchas zonas, las comunidades locales se han hecho cargo de la gestión de sus recursos culturales (Fig. 3).

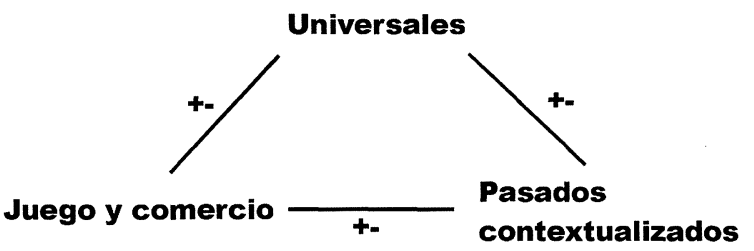

Fig. 3. Universales. Juego y comercio. Pasados contextualizados. Elementos contrapuestos de la arqueología global.

\section{CONCLUSIÓN: A FAVOR DE LA CORRIENTE}

He defendido en este artículo que la investigación arqueológica y el trabajo de campo puede ser un proceso fluido de interpretación. Puede ser reflexivo y participativo, consensuado desde perspectivas distintas. En mi opinión, esta apertura, que implica romper con las dicotomías y criticar los supuestos, se hace más necesaria en un mundo cada vez más homogéneo y diverso. Lo «políticamente correcto» se ha convertido en algo para tomarse en serio, no sólo porque esté de actualidad, sino porque se refiere a las sensibilidades y derechos de una diversidad de grupos. En arqueología ya no es posible preservar dogmáticamente las fronteras de la disciplina. Ya no es aceptable aislar 
yacimientos y equipos del mundo exterior que les rodea. Ya no es aceptable cerrar el debate diciendo «estos son los datos objetivos», puesto que otro tipo de intereses pueden querer dar sentido a los datos de forma distinta. Ya no es aceptable basar la autoridad arqueológica en un discurso cerrado, construido por los propios arqueólogos. En un mundo altamente interconectado, la autoridad tiene que defender su caso.

Para muchas personas de fines del siglo XX, acontecimientos como la guerra de Vietnam demostraron los peligros de una fe ciega en las creencias y estrategias occidentales. El descentramiento de la autoridad también ha formado parte del amplio surgimiento de voces articuladas postcoloniales. Es más, el nuevo proceso de intercambio de información global ha acelerado y desalentado a la vez la diversificación y la diferenciación.

La arqueología puede jugar un papel importante cuando estos procesos continúen y se amplíen hacia el siglo XXI. Está directamente implicada en la homogenización y diferenciación de identidades. Pero el papel que desarrolle podría ser de emancipación o de dominación. De una parte, podemos comprometernos con una arqueología multi-sitio, favoreciendo el compromiso entre los diversos intereses a todos los niveles de análisis e interpretación, desde la punta de la piqueta a la publicación final. Podemos generar una proliferación de lugares, nudos en una red global, en la que podamos reconstruir el pasado. Podemos crear chorros de pasado, un continuum de interpretación. Podemos animar a los que no están integrados en redes a que utilicen el pasado para reivindicar sus derechos e identidades o para integrarse en la red. Podemos utilizar la arqueología como un proyecto flexible, abierto y participativo con el fin de romper modelos establecidos de pensamiento y dominación. Así que, de una parte, la arqueología puede jugar un papel emancipador en el proceso global del post-colonialismo y la era de la información.

De otra parte, las mismas tecnologías que liberan y abren el debate pueden utilizarse para impedir y excluir la discusión. El flujo postmoderno de símbolos culturales (incluido el conocimiento arqueológico) puede limitarse fácilmente a una nueva élite bien informada. Los no integrados en la red están cada vez en situación de mayor desventaja en relación a los centros de dominación de los países desarrollados. Las voces alternativas se incorporan a un discurso definido y regulado desde el centro. La codificación y normalización se difunde entre los intereses del mercado de recursos culturales. Se alaba la diferencia como una parte lógica de la identificación y manipulación del mercado. Por ello, la arqueología puede jugar un papel en el proceso de dominación del ámbito globalizador.

Las nuevas tecnologías, y la flexibilidad y el pensamiento no dicotómico que permiten, no plantean una solución sino una oportunidad y un reto para los arqueólogos del nuevo milenio. Pueden utilizarse para crear emancipación en el sentido de un compromiso abierto y diverso con el pasado, una participación desde perspectivas e intereses múltiples. O pueden emplearse para excluir, cerrar y dominar. Las nuevas tecnologías, los movimientos postcoloniales y otros asuntos globales como el turismo y la preocupación por el medio ambiente, conducen a la arqueología a una nueva situación contra la que puede reaccionar cerrándose sobre sí misma o abriéndose. Puede reforzar las dicotomías o puede "dejarse llevar por la corriente". Espero que esté claro que, desde mi punto de vista, el futuro a largo plazo de la arqueología debe estar en la fluidez y las redes, más que en las dicotomías y los límites.

\section{BIBLIOGRAFÍA}

Castells, E. (1996): The rise of the Network Society. Blackwell. Oxford

Clark, G. (1957): Archaeology and society. Methuen. London. 1. ${ }^{\text {a ed. } 1939 .}$

Condori, C.M. (1989): "History and pre-history in Bolivia: what about the Indians?". In R. Layton (ed.): Conflicts in the archaeology of living traditions. Unwin Hyman. London: 46-59.

FlanNERY, K. and MARCUS, J. (1976): "Formative Oaxaca and the Zapotec Cosmos". American Scientist, 64: 374-383.

- (1993): "Cognitive archaeology". Cambridge Archaeological Journal, 3: 260-70.

FRIEDMAN, J. and Rowlands, M. (1978): The evolution of social systems. Duckworth. London.

HodDER, I. (1982): "Theoretical archaeology: a reactionary view". In I. Hodder (ed.): Symbolic and structural archaeology. Cambridge University Press. Cambridge: $1-16$.

- (1997): "Always momentary, fluid and flexible: towards a self-reflexive excavation methodology". Antiquity, 71: 691-700. 
Hunter, J. and RALSTON, I. (1993): Archaeological resource management in the UK: an introduction. Alan Sutton. Stroud.

KNAPP, A.B. and Meskell, L. (1997): "Bodies of evidence in Cypriot prehistory". Cambridge Archaeological Journal, 7 (2): 183-204.

LeEuw, S. van der and Torrence, R. (eds.) (1989): What's new?. Unwin Hyman. London.

LOWENTHAL, D. (1985): The past is a foreign country. Cambridge University Press. Cambridge.

McGuire, R. (1982): A Marxist archaeology. Academic Press. New York.

Meskell, L. (1995): "Goddesses, Gimbutas and New Age archaeology". Antiquity, 69: 74-86.

OlsEn, B. (1991): "Metropolises and satellites in archaeology". In R. Preucel (ed.): Processual and postprocessual archaeologies. Southern Illinois University, Centre for Archaeological Investigations, Occasional
Paper 10, Carbondale, Illinois (Southern Illinois University): 211-24.

PARANJPE, M. (1990): "The invasion of 'theory': an Indian response". New Quest, 81: 151-61.

RENFrEw, A.C. and BAHN, P. (1991): Archaeology. Thames and Hudson. London.

Thomas, J. (1996): Time, culture and identity. Routledge. London.

Tilley, C. (1994): The phenomenology of landscape. Berg. London.

Trigger, B. (1984): "Alternative archaeologies: nationalist, colonialist, imperialist". Man, 19: 355-70.

Uско, P. (1987): Academic freedom and apartheid: the story of the World Archaeological Congress. Duckworth. London.

- (ed.) (1995): Theory in archaeology: a world perspective. Routledge. London.

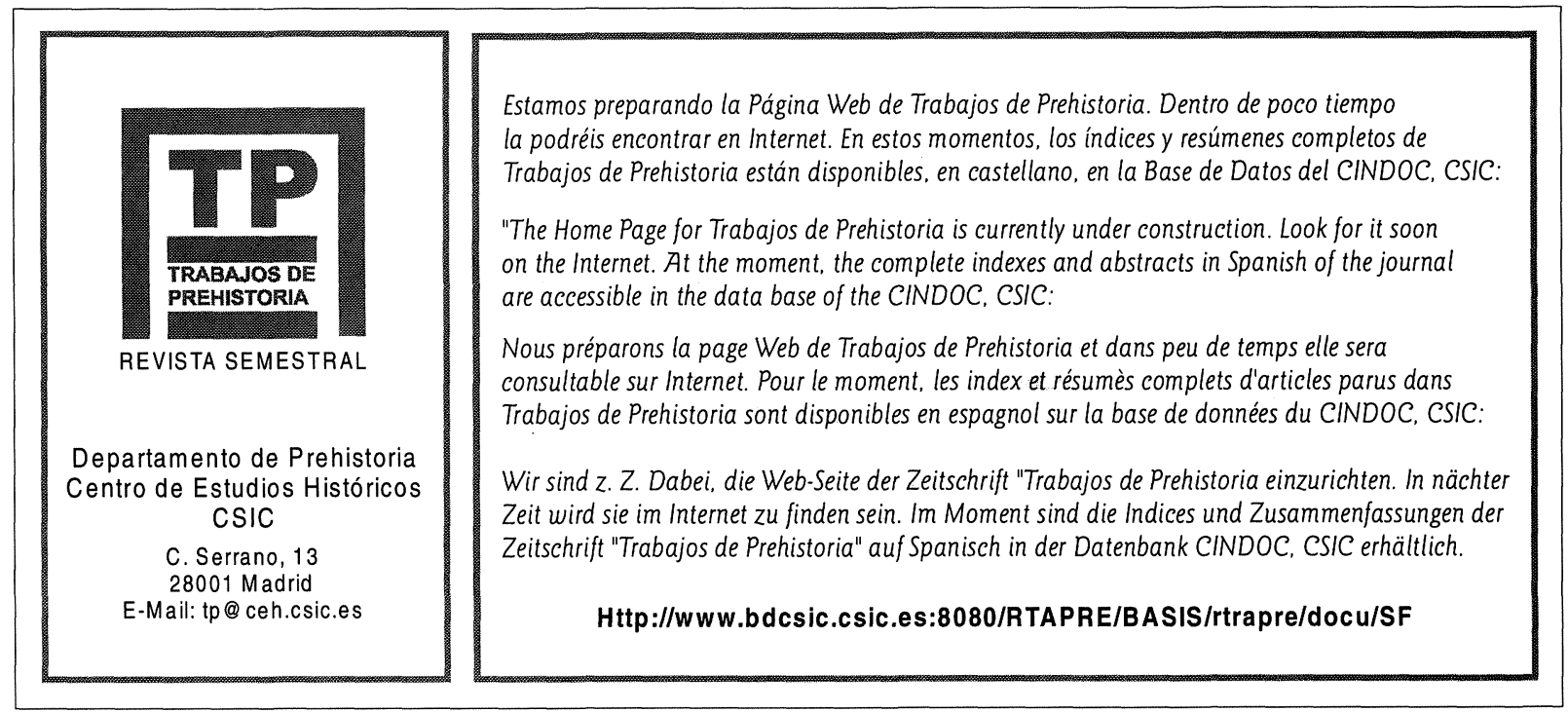

T. P., 55, n. $^{\circ} 1,1998$ 